\title{
A 2.4 GHZ WIRELESS ELECTRONIC SHIRT FOR VITAL SIGNALS MONITORING
}

\author{
J. P. Carmo ${ }^{1}$, P. M. Mendes ${ }^{2}$, C. Couto ${ }^{2}$ and J. H. Correia ${ }^{2}$ \\ ${ }^{1}$ Polytechnic Institute of Braganca, Campus Santa Apolonia, 5300, Braganca, Portugal \\ jcarmo@ipb.pt \\ ${ }^{2}$ University of Minho, Dept. Industrial Electronics, Campus Azurem, 4800-058 Guimaraes, Portugal
}

\begin{abstract}
Keywords: Wireless electronic shirt, wireless sensors networks, RF CMOS transceiver.
Abstract: $\quad$ This paper presents a wireless sensor network for wireless electronic shirts. This allows the monitoring of individual biomedical data, such as the cardio-respiratory function. The solution chosen to transmit the body's measured signals for further processing was the use of a wireless link, working at the $2.4 \mathrm{GHz}$ ISM band. A radio-frequency (RF) CMOS transceiver chip was designed in the UMC RF $0.18 \mu \mathrm{m}$ CMOS process. The power supply of the RF CMOS transceiver is of only $1.5 \mathrm{~V}$, thus it can be supplied by a single coin-sized battery. The receiver has a sensibility of $-60 \mathrm{dBm}$ and consumes $6.2 \mathrm{~mW}$. The transmitter delivers an output power of $0 \mathrm{dBm}$ with a power consumption of $15.6 \mathrm{~mW}$. Innovative topics concerning efficient power management was taken into account during the design of the RF CMOS transceiver.
\end{abstract}

\section{INTRODUCTION}

Today, the link between textiles and electronics is more realistic than ever. An emerging new field of research that combines the strengths and capabilities of electronics and textiles into one: electronic textiles, or e-textiles is opening new opportunities. E-textiles, also called smart fabrics, have not only wearable capabilities like any other garment, but also have local monitoring and computation, as well as wireless communication capabilities. Sensors and simple computational elements are embedded in e-textiles, as well as built into yarns, with the goal of gathering sensitive information, monitoring vital statistics, and sending them remotely (possibly over a wireless channel) for further processing (Marculesco et al, 2003).

The e-shirt's goal is the monitoring of the cardio-respiratory function. This makes it able to recognize qualitatively and quantitatively the presence of respiratory disorders, both during wake and sleep-time in free-living patients with chronic heart failure, providing clinical and prognostic significance data.

In wireless sensors networks, the continuous working time of sensorial nodes are limited by its average power consumption (Mackensen et al,2005). To optimise the power consumption, it was designed a RF CMOS transceiver for the operation in the $2.4 \mathrm{GHz}$ ISM band. It was used the UMC RF $0.18 \mu \mathrm{m}$ CMOS process in the design. This process has one poly and six metal layers, allowing the use of integrated spiral indutors (with a reasonable quality factor), high resistor value (a special layer is available) and the low-power supply of $1.5 \mathrm{~V}$. The use of this technology, allows the development of a complete system-on-a-chip (SOC), with the additional advantage to be supplied by a single $1.5 \mathrm{~V}$ coin-sized battery. Moreover, in order to optimize power consumption, the RF CMOS transceiver design predicts the use of control signals. With these control signals it is possible to enable and disable all the subsystems of the transceiver. These signals allows, e.g., to switch off the receiver when a RF signal is being transmitted, to switch off the transmitter when a RF signal is being received, and allows the transceiver to enter to sleep when RF signals are neither being transmitted, nor being received.

\section{TRANSCEIVER DESIGN}

Figure 1 shows the architecture of the RF CMOS transceiver, which consists of a receiver, a 
transmitter, an antenna-switch and a frequency synthesizer.

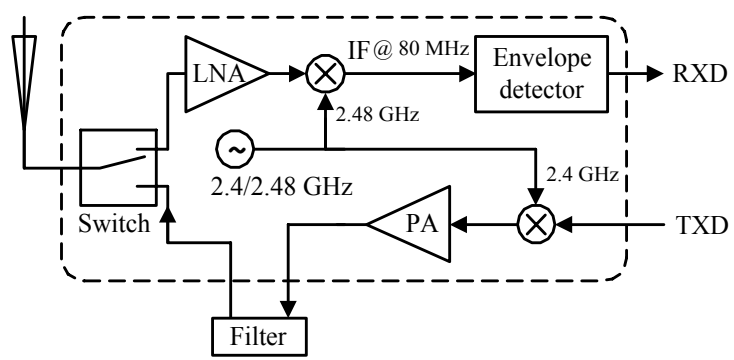

Figure 1: The block diagram of the RF CMOS transceiver.

The receiver adopts a direct demodulation, by means of heterodyne detection. It is enough to achieve a bit error probability less that $10^{-6}$ with a sensibility of $-60 \mathrm{dBm}$, in a transmitted power of $0 \mathrm{dBm}$ using Amplitude Shift Keying (ASK) modulation. These specifications makes the RF CMOS transceiver suitable for short range applications.

\subsection{Receiver}

Figure 2 shows the receiver's front-end schematic. The amplified RF signal at the output of the low-noise amplifier (LNA) is directly converted to an intermediate frequency (IF) with a double-balanced downconversion mixer, followed by a low-pass filtering. The final downconversion to the baseband is made by means of envelope detection.

The low-noise amplifier (LNA) is the first gain stage in the receiver path. In a LNA, the signal must be amplified as much as possible, with a small signal-to-noise ratio (SNR) decrease. This is achieved with the best noise figure (NF). The LNA is an inductively degenerated common source amplifier. This makes the input impedance at $2.4 \mathrm{GHz}$ equal to $50 \Omega$, for matching with antenna-switch (Yao, 2007). The LNA is putted in the sleeping mode, by cutting the current in the polarization stage. The same principle applies to the all subsystems of the transceiver. The downconversion to the IF uses a double-balanced switched transconductor mixer (Klumperink, 2004). The advantage is that the switching stage is directly connected to the power rails, requiring a less voltage headroom. This simplifies the switching process and has a slightly bigger conversion gain. Moreover, this topology also eliminates the presence of the strong carrier at the output, producing only odd harmonics of the local oscillation frequency. The signal at the output of the low-pass filter enters an envelope detector. The $80-\mathrm{MHz}$ IF frequency is produced from the $2.4 \mathrm{GHz} \mathrm{RF}$ and from the $2.48 \mathrm{GHz}$ local frequency.

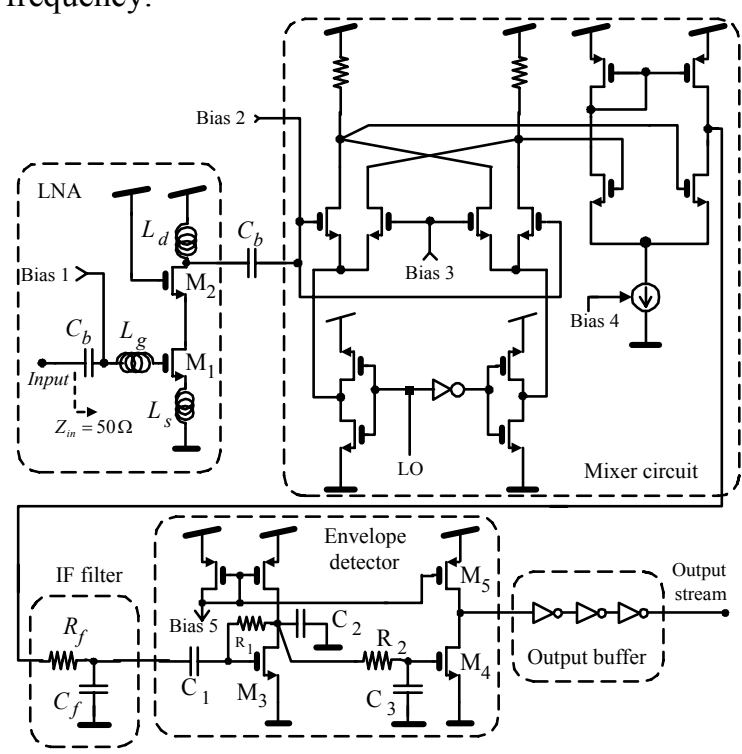

Figure 2: The schematic of the receiver.

\subsection{Transmitter}

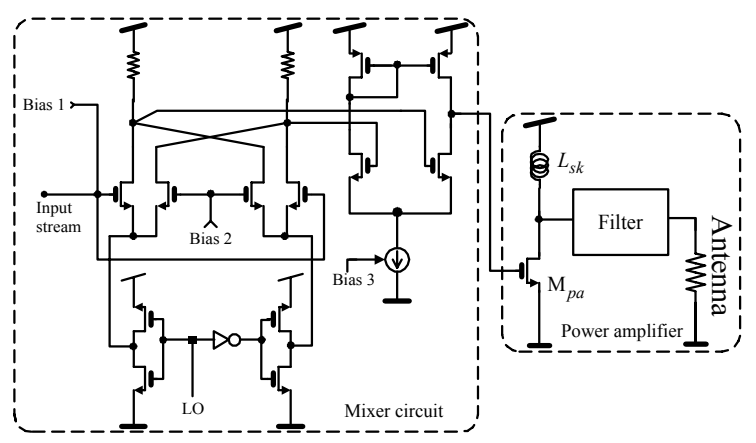

Figure 3: The schematic of the transmitter.

Figure 3 shows the schematic of the RF CMOS transmitter. The mixer used in the frequency upconversion is of the same type of the previous mixer used in the frequency downconversion. The main differences are on the MOSFET's sizes. The $\mathrm{RF}$ signal is further amplified by a power amplifier, which provides a transmitted signal with an appropriated output power. The upconversion mixer generates the RF signal, from the input bitstream and from a $2.4 \mathrm{GHz}$ local generated frequency. The external filter follows the power amplifier, and removes the spectral components around the $2.4 \mathrm{GHz}$ carrier frequency while adapts it to the impedance of the antenna. 


\subsection{Frequency Synthesizer}

As depicted in Figure 4, the PLL has a reference generator circuit with a crystal based oscillator at $20 \mathrm{MHz}$, followed by a phase-frequency difference circuit (PFD) without dead zone, a current steering charge pump (CP) and a third order passive filter. The passive section output is connected to the VCO, which generates the desired frequencies of $2.4 \mathrm{GHz}$ or $2.48 \mathrm{GHz}$. These frequencies must be divided by 120 or by 124 and connected to the PFD again, closing the loop. The frequency division ratio is selected by the one bit control signal $120 / 124$. This selects the value of the frequency to be connected to the mixer, in order to make the up or the down-conversion operation.

The division by $120 / 124$ in the feedback path is done with a cascade constituted by one half divider implemented with a true single phase clock (TSPC) logic, one divider by $30 / 31$ (digitally selected by the signal 120/124), followed by a toggle flip-flop to ensure a duty-cycle of $50 \%$ at the PFD input. The TSPC logic was used to overcome the impossibility to implement the first toggle flip-flop with static logic in this technology. It is required a rail-to-rail input to work properly. Moreover, at these frequencies, the power consumptions are lowest compared with the SCL logic (Pellerano et al, 2004). The ratios of 30 and 31 were achieved with the use of frequency dividers by $2 / 3$ with modulus control.

\section{WIRELESS ELECTRONIC SHIRT}

Like any other every-day garment piece, the wireless electronic shirt (WES) will be lightweight, machine washable, comfortable, easy-to-use shirt with embedded sensors. To measure respiratory and cardiac functions, sensors are plugged into the shirt around patient's chest and abdomen. The WES also uses small sizes and compact modules, made with microsystems, containing the $2.4 \mathrm{GHz} \mathrm{RF}$ CMOS transceiver, the electronic of control and processing, and the interfaces to make the connections to the sensors. These modules have also an associated antenna and are supplied by a coin-sized battery. The size-reduction achieved with these modules, make them suitable to be easily plugged in the WES, according the interest of the medical doctors.

Conventional applications of electronic shirts uses wires to connect the sensors to a central unit, and then to make the RF communication to the external base-station (Marculescu et al., 2003). An interesting application and an easy way to implement wireless buses, is using modules able to communicate between them and between the base-station.

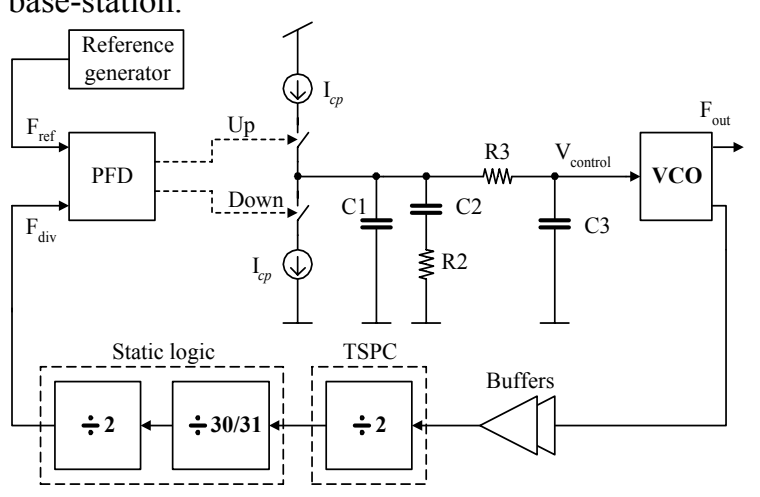

Figure 4: The structure of the PLL.

A single channel measures heart rate and an inductive elastic band will be used for monitoring the respiratory function.

It is used a bending sensor to monitor the shoulders positions, that suffers changes in the electrical resistance when it is bent. As this sensor is bent the resistance gradually increases. When the sensor is bent at 90 degrees, its resistance will range between from the $30 \mathrm{k} \Omega$ up to the $40 \mathrm{k} \Omega$. It was also used an elastic band to measure the changes in thoracic circumference due to respiration. The transducer contains an variable inductance, used indirectly to measure the changes in thoracic circumference. The device should be placed around the body at the level of maximum respiratory expansion. This level will change between erect and supine positions. At maximum inspiration the belt should be stretched almost to maximum extension, making its inductance minimum. As shown in the Figure 5, the PLL reference oscillator is reused, to generate the $20 \mathrm{MHz}$ sinusoidal signal, required for this circuit. The variations in the inductance, changes the attenuation for the $20 \mathrm{MHz}$ signal, in the first $R L$ low-pass of first order filter. Thus, the attenuation increases with the decreasing of the thorax perimeter. This filtered signal is further amplified, before a second low-pass filtering, to eliminate noise and spurs generated in the $20 \mathrm{MHz}$ oscillator. Then, a peak detector gets the amplitude of the $20 \mathrm{MHz}$ processed signal. This amplitude is a low-frequency signal, and it is converted to the digital domain, using a $\Sigma \Delta$ analog-to-digital converter (ADC) of first order, with an output with resolution of eight bits. This allows the recording of respiratory changes with maximum sensitivity and linearity. 


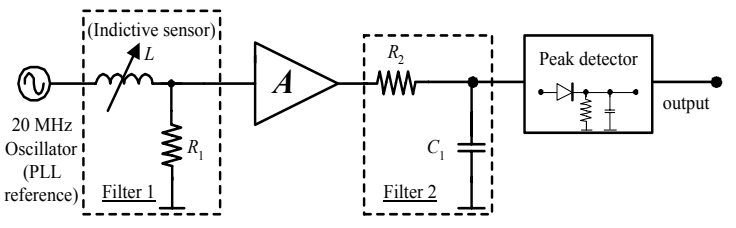

Figure 5: The block diagram of a signal conditioning used in the measure of the respiratory function.

It is shown in Figure 6, a photo of the patient wearing an WES ready to plug the RF modules. It can be seen the three connections for heart-rate respiratory function.

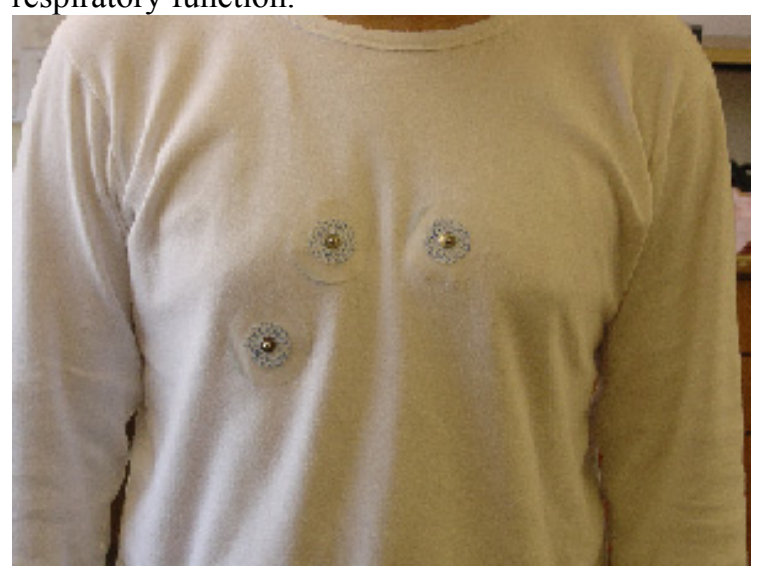

Figure 6: A photo of the patient wearing a electronic shirt ready to plug the RF modules.

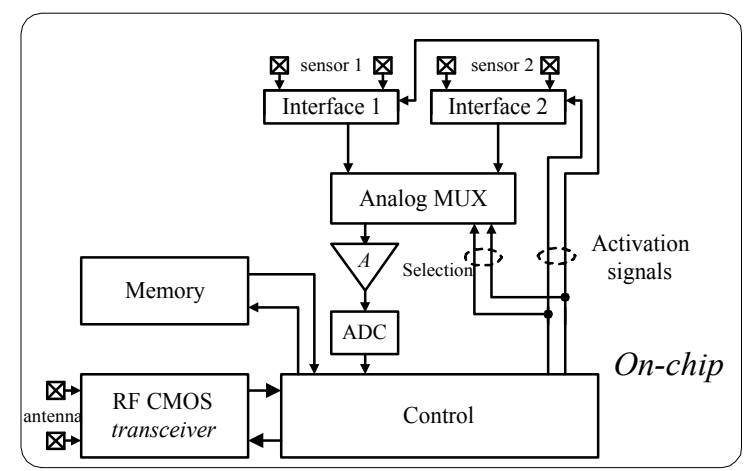

Figure 7: Block diagram of the microsystems used in the plug-and-play modules of the WES.

In WES, the sensor interfaces, data processing, the wireless interface and antenna are integrated in the same microsystem (Figure 7) by Multi-Chip-Module (MCM) techniques. The wireless communication is between the base-station and the multiple processing elements placed in the shirt. The main advantage is to allow the positioning of the sensors where we like. The sensors can also be removed from the shirt, either when the sensors are no more need or when the shirt is to be cleaned and washed. This wireless bus introduces the concept of plug-and-play in textiles.

\section{CONCLUSIONS}

The simulations of the RF CMOS transceiver, shown a total power consumption of $6.2 \mathrm{~mW}$ for the receiver $(1.5 \mathrm{~mW}$ for the LNA $3.2 \mathrm{~mW}$ for the downconversion mixer, and $1.5 \mathrm{~mW}$ for the envelope detector). For the transmitter, it was observed the power consumptions in the following blocks: $5.3 \mathrm{~mW}$ in the upconversion mixer and $10.3 \mathrm{~mW}$ in the PA. The transmitter delivers a maximum output power of $1.08 \mathrm{~mW}$ with a total power consumption of $11.2 \mathrm{~mW}$. These characteristics fulfill the requirements for shortrange communications for using the $2.4 \mathrm{GHz}$ ISM band.

The main goal of the WES, is improving the monitoring of the cardio-respiratory function, by using devices which reduces healthcare costs and facilitates the diagnostic while at the same time preserving the mobility and lifestyle of patients.

\section{REFERENCES}

Mackensen, E., et al, 2005, Enhancing the lifetime of autonomous microsystems in wireless sensor actuator networks (WSANs), in Proceedings of the XIX Eurosensors, TC4, pp.1-2, Barcelona, Spain.

Klumperink, E., et al, 2004, A CMOS switched transconductor mixer, IEEE Journal of Solid-State Circuits, Vol. 39, No. 8, pp. 1231-1240.

Marculescu, D., et al., 2003, Electronic Textiles: A Platform for Pervasive Computing, Proceedings of the IEEE, Vol. 91, No. 12, pp. 1995-2018.

Pellerano, S., et al, 2004, A $13.5 \mathrm{~mW}$ 5-GHz frequency sinthesizer with dynamic logic frequency divider, IEEE Journal of Solid-State Circuits, Vol. 39, No. 2, pp. 378-383.

Yao, T., et al, 2007, Algorithmic design of CMOS LNAs and PAs for $60-\mathrm{GHz}$ radio, IEEE Journal of Solid-State Circuits, Vol. 42, No. 5, pp. 1044-1057. 
MAIN AUTHOR VITAE

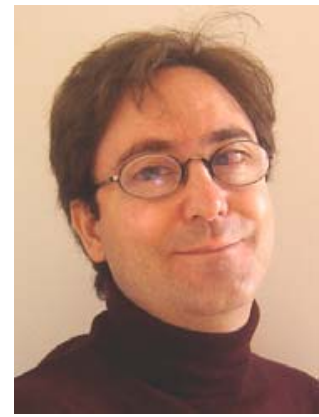

J.P. Carmo graduated in 1993 and received his MSc degree in 2002, both in Electrical Engineering and Computers from the University of Oporto, Oporto, Portugal. In 2007, he obtained the PhD degree in Industrial Electronics at the University of Minho, Portugal.

From 1999 to May 2008, he was a lecturer at the Polytechnic Institute of Bragança, Portugal.

Currently, he is currently involved and the leader in projects, concerning the research on RF applications and microsystems, at the Algoritmi center at University of Minho, Guimarães, Portugal. 